SFB

Nonparametric change-point tests for long-range dependent data

Herold Dehling, Aeneas Rooch, Murad S. Taqqu

Nr. 27/2011
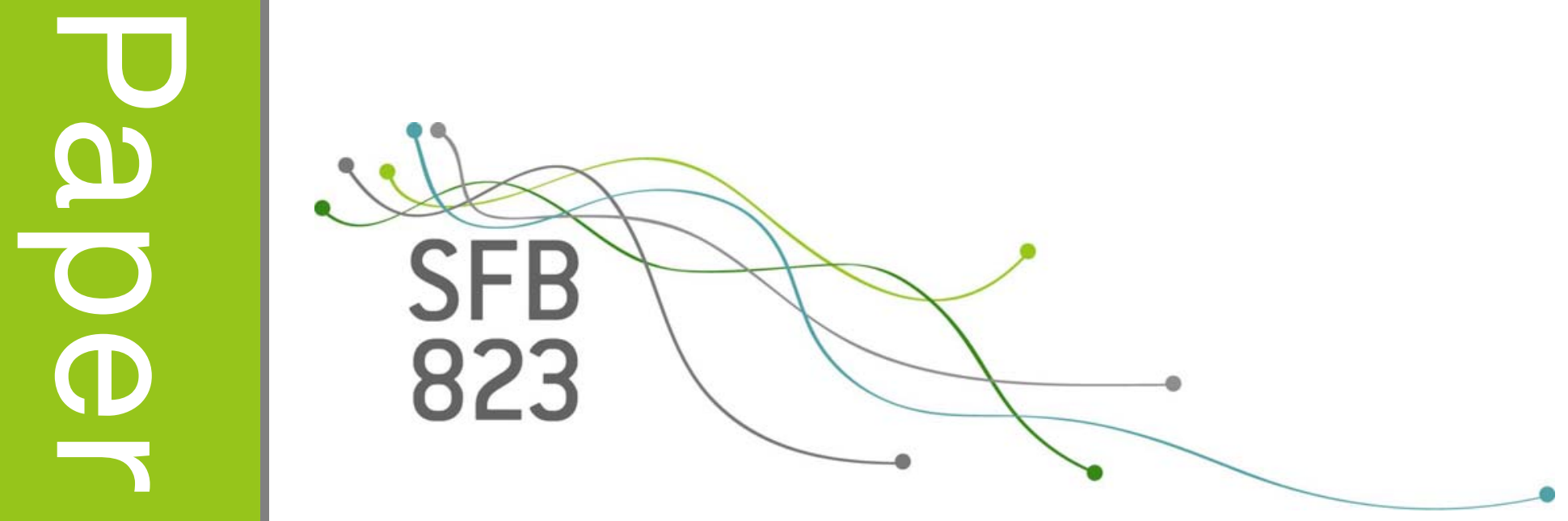

August 4, 2011

\title{
NONPARAMETRIC CHANGE-POINT TESTS FOR LONG-RANGE DEPENDENT DATA
}

\author{
HEROLD DEHLING, AENEAS ROOCH, AND MURAD S. TAQQU
}

\begin{abstract}
We propose a nonparametric change-point test for long-range dependent data, which is based on the Wilcoxon two-sample test. We derive the asymptotic distribution of the test statistic under the null-hypothesis that no change occured. In a simulation study, we compare the power of our test with the power of a test which is based on differences of means. The results of the simulation study show that in the case of Gaussian data, our test has only slightly smaller power than the difference-of-means test. For heavy-tailed data, our test outperforms the difference-of-means test.
\end{abstract}

\section{Introduction and Statement of Main Results}

In this paper we study change-point problems in long-range dependent time series. Specifically we consider the model where the observations are generated by a stochastic process $\left(X_{i}\right)_{i \geq 1}$ of the type

$$
X_{i}=\mu_{i}+\epsilon_{i},
$$

where $\left(\epsilon_{i}\right)_{i \geq 1}$ is a long-range dependent stationary process with mean zero, and where $\left(\mu_{i}\right)_{i \geq 1}$ are the unknown means. We focus on the case when $\left(\epsilon_{i}\right)_{i \geq 1}$ is an instantaneous functional of a stationary Gaussian process with non-summable covariances, i.e.

$$
\epsilon_{i}=G\left(\xi_{i}\right), \quad i \geq 1 .
$$

We assume that $\left(\xi_{i}\right)_{i \geq 1}$ is a mean-zero Gaussian process with $E\left(\xi_{i}^{2}\right)=1$ and long-range dependence, that is, with autocovariance function

$$
\rho(k)=k^{-D} L(k), \quad k \geq 1,
$$

where $0<D<1$, and where $L(k)$ is a slowly varying function. Moreover, $G: \mathbb{R} \rightarrow \mathbb{R}$ is a measurable function satisfying $E\left(G\left(\xi_{i}\right)\right)=0$.

Based on observations $X_{1}, \ldots, X_{n}$, we wish to test the hypothesis

$$
H: \mu_{1}=\ldots=\mu_{n}
$$

that there is no change in the means of the data against the alternative

$$
A: \mu_{1}=\ldots=\mu_{k} \neq \mu_{k+1}=\ldots=\mu_{n} \text { for some } k \in\{1, \ldots, n-1\} .
$$

We shall refer to this test problem as $(H, A)$.

In the case of independent observations, change-point problems have been much investigated, and both parametric as well as nonparametric tests have been studied. For an excellent survey, see the monograph by Csörgö and Horváth (1997). There are also many results for weakly dependent observations. E.g., Ling (2007) studies the asymptotic distribution of the Wald test for parameter changes in near epoch dependent processes. Aue, Hörmann, Horváth and Reimherr (2009) study tests for detecting breaks in the covariance

Key words and phrases. Change-point problems, nonparametric change-point tests, Wilcoxon two-sample rank test, long-range dependent data, long memory, functional limit theorem.

Herold Dehling and Aeneas Rooch were supported in part by the Collaborative Research Grant 823, Project C3 Analysis of Structural Change in Dynamic Processes, of the German Research Foundation. Murad S. Taqqu was supported in part by the NSF grants DMS-0608669 and DMS-1007616 at Boston University. 
structure of multivariate time series. Wied, Krämer and Dehling (2011) study change-points in the correlation structure of near epoch dependent processes.

In the case of long-range dependent data, much less is known. Csörgő and Horváth (1997) study so-called cusum tests for changes in the mean, which are based on statistics of the type

$$
\max _{1 \leq k \leq n}\left|\frac{1}{k} \sum_{i=1}^{k} X_{i}-\frac{1}{n} \sum_{i=1}^{n} X_{i}\right|
$$

Krämer, Sibbertsen and Kleiber (2002) observe that the presence of long-range dependence might lead to false rejection of the hypothesis of stationarity in change-point tests. Berkes, Horváth, Kokoszka and Shao (2006) propose cusum type tests for discriminating between long-range dependence and changes in the mean of a weakly dependent process.

In the present paper we want to investigate rank tests for changes in the mean. Antoch, Hušková, Janic and Ledwina (2008) studied rank tests for i.i.d. observations. Wang (2008) investigated rank tests for change-points in long-range dependent linear processes. In this paper, we study rank tests for changes in the mean of long-range dependent processes that have a representation as instantaneous nonlinear functionals of stationary Gaussian processes.

Tests for change-point problems usually start from the two-sample problem that one obtains when the change-point is regarded as being known, that is, where for a given $k \in\{1, \ldots, n-1\}$, the alternative is

$$
A_{k}: \mu_{1}=\ldots=\mu_{k} \neq \mu_{k+1}=\ldots=\mu_{n} .
$$

For the test problem $\left(H, A_{k}\right)$, the Wilcoxon two-sample rank test is a commonly used nonparametric test. The Wilcoxon test rejects for large and small values of the test statistic

$$
W_{k, n}=\sum_{i=1}^{k} \sum_{j=k+1}^{n} 1_{\left\{X_{i} \leq X_{j}\right\}}
$$

$W_{k, n}$ counts the number of times the second part of the sample exceeds the first part of the sample. Note that this is the Mann-Whitney U-statistic representation of Wilcoxon's rank test statistic; see e.g. Lehmann (1975).

Tests for the change-point problem $(H, A)$, where one does not suppose that $k$ is known, can be based on the vector of test statistics $W_{1, n}, \ldots, W_{n-1, n}$. In a parametric model, the generalized likelihood ratio test rejects for large values of the maximum of the test statistics obtained for the two-sample problems $\left(H, A_{k}\right)$; see e.g. Csörgö and Horvath (1997). Also in the nonparametric case, a common procedure is to reject the null hypothesis for large values of $W_{n}=\max _{k=1, \ldots, n-1}\left|W_{k, n}\right|$, though one could in principle take other functions of $W_{1, n}, \ldots, W_{n-1, n}$.

In order to set the critical values of the test, we need to know the distribution of $W_{n}$ under the null hypothesis of no change. Since the exact distribution is hard to obtain, we consider the asymptotic distribution for a large sample size. To this end we consider the process

$$
W_{n,[n \lambda]}=\sum_{i=1}^{[n \lambda]} \sum_{j=[n \lambda]+1}^{n} 1_{\left\{X_{i} \leq X_{j}\right\}}, 0 \leq \lambda \leq 1,
$$

parametrized by $\lambda$. After centering and normalizing, we obtain the process

$$
W_{n}(\lambda)=\frac{1}{n d_{n}} \sum_{i=1}^{[n \lambda]} \sum_{j=[n \lambda]+1}^{n}\left(1_{\left\{X_{i} \leq X_{j}\right\}}-\int_{\mathbb{R}} F(x) d F(x)\right), 0 \leq \lambda \leq 1 .
$$


Observe that the centering constant $\int F(x) d F(x)$ equals $E\left(1_{\left\{X_{1} \leq X_{1}^{\prime}\right\}}\right)$, where $X_{1}^{\prime}$ is an independent copy of $X_{1}$. The latter is the proper normalization as the dependence between $X_{i}$ and $X_{j}$ vanishes asymptotically when $|j-i| \rightarrow \infty$. If the distribution function $F$ is continuous, we get $\int_{\mathbb{R}} F(x) d F(x)=\frac{1}{2}$. Note that under the null hypothesis, the distribution of $W_{n}(\lambda)$ does not depend on the common mean $\mu:=\mu_{1}=\ldots=\mu_{n}$. Thus, we may assume without loss of generality that $\mu=0$ and hence that $X_{i}=G\left(\xi_{i}\right)$.

In order to analyze the asymptotic distribution of the process $\left(W_{n}(\lambda)\right)_{0 \leq \lambda \leq 1}$, we apply the empirical process invariance principle of Dehling and Taqqu (1989) to the sequence $\left(G\left(\xi_{i}\right)\right)_{i \geq 1}$. We consider the Hermite expansion

$$
1_{\left\{G\left(\xi_{i}\right) \leq x\right\}}-F(x)=\sum_{q=1}^{\infty} \frac{J_{q}(x)}{q !} H_{q}\left(\xi_{i}\right),
$$

where $H_{q}$ is the $q$-th order Hermite polynomial and where

$$
J_{q}(x)=E H_{q}\left(\xi_{i}\right) 1_{\left\{G\left(\xi_{i}\right) \leq x\right\}} .
$$

We define the Hermite rank of the class of functions $\left\{1_{\left\{G\left(\xi_{i}\right) \leq x\right\}}-F(x), x \in \mathbb{R}\right\}$, by

$$
m:=\min \left\{q \geq 1: J_{q}(x) \neq 0 \text { for some } x \in \mathbb{R}\right\},
$$

and the normalizing constants

$$
d_{n}^{2}=\operatorname{Var}\left(\sum_{j=1}^{n} H_{m}\left(\xi_{j}\right)\right)
$$

We make the further assumption that $0<D<\frac{1}{m}$, in which case

$$
d_{n}^{2} \sim c_{m} n^{2-m D} L^{m}(n),
$$

where $c_{m}=\frac{2 m !}{(1-D m)(2-D m)}$. Here we use the symbol $a_{n} \sim b_{n}$ to denote that $a_{n} / b_{n} \rightarrow 1$ as $n \rightarrow \infty$. Then by the two-parameter empirical process invariance principle of Dehling and Taqqu (1989),

$$
\left(d_{n}^{-1}[n \lambda]\left(F_{[n \lambda]}(x)-F(x)\right)\right)_{x \in[-\infty, \infty], \lambda \in[0,1]} \rightarrow\left(\frac{J_{m}(x)}{m !} Z_{m}(\lambda)\right)_{x \in[-\infty, \infty], \lambda \in[0,1]},
$$

weakly in $D([-\infty, \infty] \times[0,1])$, where $\left(Z_{m}(\lambda)\right)_{\lambda \in[0,1]}$ is an $m$-th order Hermite process, defined in Taqqu (1979), formula (1.3). A spectral representation of $Z_{m}$ is given in formula (1.7) of Dehling and Taqqu (1989). Observe the separation of variables in the limit: the limiting process $Z_{m}(x, \lambda)$ is expressible as $\frac{J_{m}(x)}{m !} Z_{m}(\lambda)$.

The process $\left(Z_{m}(\lambda)\right)_{\lambda \geq 0}$ is self-similar with parameter

$$
H=1-\frac{m D}{2} \in\left(\frac{1}{2}, 1\right),
$$

that is, $Z_{m}(c \lambda)$ and $c^{H} Z_{m}(\lambda)$ have the same finite-dimensional distributions for all constants $c>0 .{ }^{1}$ The process $Z_{m}(\lambda)$ is not Gaussian when $m \geq 2$. When $m=1, Z_{1}(\lambda)$ is the standard Gaussian fractional Brownian motion, often denoted $B_{H}(\lambda)$. It is Gaussian with mean zero and covariance

$$
E\left(Z_{1}\left(\lambda_{1}\right) Z_{1}\left(\lambda_{2}\right)\right)=\frac{1}{2}\left\{\lambda_{1}^{2 H}+\lambda_{2}^{2 H}-\left|\lambda_{1}-\lambda_{2}\right|^{2 H}\right\}
$$

\footnotetext{
${ }^{1}$ Do not confuse the index $H$, which is called Hurst parameter with the other $H$ 's used in this paper to denote hypothesis and Hermite polynomial
} 
Theorem 1.1. Suppose that $\left(\xi_{i}\right)_{i \geq 1}$ is a stationary Gaussian process with mean zero, variance 1 , and autocovariance function as in (1) with $0<D<\frac{1}{m}$. Moreover let $G: \mathbb{R} \rightarrow \mathbb{R}$ be a measurable function and define

$$
X_{k}=G\left(\xi_{k}\right) \text {. }
$$

Assume that $X_{k}$ has a continuous distribution function $F$. Let $m$ denote the Hermite rank of the class of functions $1_{\left\{G\left(\xi_{i}\right) \leq x\right\}}-F(x), x \in \mathbb{R}$, as defined in (3) and let $d_{n}>0$ satisfy (5). Then

$$
\frac{1}{n d_{n}} \sum_{i=1}^{[n \lambda]} \sum_{j=[n \lambda]+1}^{n}\left(1_{\left\{X_{i} \leq X_{j}\right\}}-\int_{\mathbb{R}} F(x) d F(x)\right), \quad 0 \leq \lambda \leq 1,
$$

converges in distribution towards the process

$$
\frac{1}{m !}\left(Z_{m}(\lambda)-\lambda Z_{m}(1)\right) \int_{\mathbb{R}} J_{m}(x) d F(x), \quad 0 \leq \lambda \leq 1 .
$$

\section{Proof of TheOREM 1.1}

We introduce the empirical distribution functions of the first $k$ and the last $(n-k)$ observations, respectively:

$$
\begin{aligned}
F_{k}(x) & =\frac{1}{k} \sum_{i=1}^{k} 1_{\left\{X_{i} \leq x\right\}} \\
F_{k+1, n}(x) & =\frac{1}{n-k} \sum_{i=k+1}^{n} 1_{\left\{X_{i} \leq x\right\}}
\end{aligned}
$$

so that $[n \lambda] F_{[n \lambda]}(x)=\sum_{i=1}^{[n \lambda]} 1_{\left\{X_{i} \leq x\right\}}$ and $(n-[n \lambda]) F_{[n \lambda]+1, n}=\sum_{i=[n \lambda]+1}^{n} 1_{\left\{X_{i} \leq x\right\}}$. Then we obtain the following representation

$$
\begin{aligned}
& \sum_{i=1}^{[n \lambda]} \sum_{j=[n \lambda]+1}^{n}\left(1_{\left\{X_{i} \leq X_{j}\right\}}-\int_{\mathbb{R}} F(x) d F(x)\right) \\
&= {[n \lambda] \sum_{j=[n \lambda]+1}^{n}\left(F_{[n \lambda]}\left(X_{j}\right)-\int_{\mathbb{R}} F(x) d F(x)\right) } \\
&=[n \lambda](n-[n \lambda])\left(\int_{\mathbb{R}} F_{[n \lambda]}(x) d F_{[n \lambda]+1, n}(x)-\int_{\mathbb{R}} F(x) d F(x)\right) \\
&=[n \lambda](n-[n \lambda]) \int_{\mathbb{R}}\left(F_{[n \lambda]}(x)-F(x)\right) d F_{[n \lambda]+1, n}(x) \\
& \quad+[n \lambda](n-[n \lambda]) \int_{\mathbb{R}} F(x) d\left(F_{[n \lambda]+1, n}-F\right)(x) \\
&=[n \lambda](n-[n \lambda]) \int_{\mathbb{R}}\left(F_{[n \lambda]}(x)-F(x)\right) d F_{[n \lambda]+1, n}(x) \\
& \quad-[n \lambda](n-[n \lambda]) \int_{\mathbb{R}}\left(F_{[n \lambda]+1, n}(x)-F(x)\right) d F(x),
\end{aligned}
$$

where in the third line we used the relation $E(K(X))=\int_{\mathbb{R}} K(x) d F(x)$, where $F$ and $E$ are respectively, the empirical distribution and the empirical mean of $X$, and where, in the final step, we have used integration by parts, namely $\int_{\mathbb{R}} G d F=1-\int_{\mathbb{R}} F d G$, if $F$ and $G$ are 
two distribution functions. We now apply the empirical process non-central limit theorem of Dehling and Taqqu (1989) which states that

$$
\left(d_{n}^{-1}[n \lambda]\left(F_{n \lambda}(x)-F(x)\right)\right)_{x \in[-\infty, \infty], \lambda \in[0,1]} \stackrel{\mathcal{D}}{\longrightarrow}(J(x) Z(\lambda))_{x \in[-\infty, \infty], \lambda \in[0,1]},
$$

where

$$
J(x)=J_{m}(x) \quad \text { and } \quad Z(\lambda)=Z_{m}(\lambda) / m ! .
$$

By the Dudley-Wichura version of Skorohod's representation theorem (see Shorack and Wellner (1986), Theorem 2.3.4) we may assume without loss of generality that convergence holds almost surely with respect to the supremum norm on the function space $D([0,1] \times[-\infty, \infty])$, i.e.

$$
\sup _{\lambda, x}\left|d_{n}^{-1}[n \lambda]\left(F_{n \lambda}(x)-F(x)\right)-J(x) Z(\lambda)\right| \longrightarrow 0 \quad \text { a.s. }
$$

Note that, by definition,

$$
(n-[n \lambda])\left(F_{[n \lambda]+1, n}(x)-F(x)\right)=n\left(F_{n}(x)-F(x)\right)-[n \lambda]\left(F_{[n \lambda]}(x)-F(x)\right) .
$$

Applying (9) to each of the terms on the right-hand side of (10), we obtain the following limit theorem for the two parameter empirical process of the observations $X_{[n \lambda]+1}, \ldots, X_{n}$.

$$
\sup _{\lambda, x}\left|d_{n}^{-1}(n-[n \lambda])\left(F_{[n \lambda]+1, n}(x)-F(x)\right)-J(x)(Z(1)-Z(\lambda))\right| \longrightarrow 0 .
$$

Thus we get, for the first term in the right-hand side of (8),

$$
\begin{aligned}
& \frac{1}{n d_{n}}[n \lambda](n-[n \lambda]) \int_{\mathbb{R}}\left(F_{[n \lambda]}(x)-F(x)\right) d F_{[n \lambda]+1, n}(x)-(1-\lambda) \int_{\mathbb{R}} J(x) Z(\lambda) d F(x) \\
& =\frac{n-[n \lambda]}{n} \int_{\mathbb{R}} d_{n}^{-1}[n \lambda]\left(F_{[n \lambda]}(x)-F(x)\right) d F_{[n \lambda]+1, n}(x)-\frac{n-[n \lambda]}{n} \int_{\mathbb{R}} J(x) Z(\lambda) d F(x) \\
& \quad+\left\{\frac{n-[n \lambda]}{n}-(1-\lambda)\right\} \int_{\mathbb{R}} J(x) Z(\lambda) d F(x) \\
& =\frac{n-[n \lambda]}{n} \int_{\mathbb{R}}\left\{d_{n}^{-1}[n \lambda]\left(F_{[n \lambda]}(x)-F(x)\right)-J(x) Z(\lambda)\right\} d F_{[n \lambda]+1, n}(x) \\
& \quad+\frac{n-[n \lambda]}{n} \int_{\mathbb{R}} J(x) Z(\lambda) d\left(F_{[n \lambda]+1, n}-F\right)(x) \\
& \quad+\left\{\frac{n-[n \lambda]}{n}-(1-\lambda)\right\} \int_{\mathbb{R}} J(x) Z(\lambda) d F(x) .
\end{aligned}
$$

The first term on the right-hand side converges to 0 almost surely, by (9). The third term converges to zero as

$$
\sup _{0 \leq \lambda \leq 1}\left|\frac{n-[n \lambda]}{n}-(1-\lambda)\right| \rightarrow 0 .
$$

Regarding the second term, note that $\int_{\mathbb{R}} J(x) d F(x)=E\left(J\left(X_{i}\right)\right)$ and hence

$$
\begin{aligned}
& \frac{n-[n \lambda]}{n} \int_{\mathbb{R}} J(x) Z(\lambda) d\left(F_{[n \lambda]+1, n}-F\right)(x) \\
& =Z(\lambda) \frac{1}{n} \sum_{i=[n \lambda]+1}^{n}\left(J\left(X_{i}\right)-E J\left(X_{i}\right)\right) \\
& =Z(\lambda)\left\{\frac{1}{n} \sum_{i=1}^{n}\left(J\left(X_{i}\right)-E\left(J\left(X_{i}\right)\right)\right)-\frac{1}{n} \sum_{i=1}^{[n \lambda]}\left(J\left(X_{i}\right)-E\left(J\left(X_{i}\right)\right)\right)\right\} .
\end{aligned}
$$


By the ergodic theorem, $\frac{1}{n} \sum_{i=1}^{n}\left(J\left(X_{i}\right)-E\left(J\left(X_{i}\right)\right)\right) \rightarrow 0$, almost surely. Hence $\sum_{i=1}^{n}\left(J\left(X_{i}\right)-\right.$ $\left.E\left(J\left(X_{i}\right)\right)\right)=o(n)$ and thus

$$
\max _{1 \leq k \leq n}\left|\sum_{i=1}^{k}\left(J\left(X_{i}\right)-E\left(J\left(X_{i}\right)\right)\right)\right|=o(n), \text { as } n \rightarrow \infty, \quad \text { a.s. }
$$

Hence (12) converges to zero almost surely, uniformly in $\lambda \in[0,1]$.

Regarding the second term on the right-hand side of (8) we obtain

$$
\begin{aligned}
& \frac{1}{n d_{n}}[n \lambda](n-[n \lambda]) \int_{\mathbb{R}}\left(F_{[n \lambda]+1, n}-F(x)\right) d F(x)-\lambda \int_{\mathbb{R}} J(x)(Z(1)-Z(\lambda)) d F(x) \\
& =\frac{[n \lambda]}{n} \int_{\mathbb{R}}\left\{d_{n}^{-1}(n-[n \lambda])\left(F_{[\lambda n]+1, n}(x)-F(x)\right)-J(x)(Z(1)-Z(\lambda))\right\} d F(x) \\
& \quad-\left(\lambda-\frac{[n \lambda]}{n}\right) \int_{\mathbb{R}} J(x)(Z(1)-Z(\lambda)) d F(x) .
\end{aligned}
$$

Both terms on the right-hand side converge to zero a.s., uniformly in $\lambda \in[0,1]$. For the first term, this follows from (11). For the second term, this holds since $\sup _{0 \leq \lambda \leq 1}\left|\frac{[n \lambda]}{n}-\lambda\right| \rightarrow 0$, as $n \rightarrow \infty$. Using (8) and the fact that the right-hand sides of (12) and (13) converge to zero uniformly in $0 \leq \lambda \leq 1$, we have proved that the normalized Wilcoxon two-sample test statistic (7) converges in distribution towards

$$
\int_{\mathbb{R}}(1-\lambda) Z(\lambda) J(x) d F(x)-\int_{\mathbb{R}} \lambda(Z(1)-Z(\lambda)) J(x) d F(x), \quad 0 \leq \lambda \leq 1 .
$$

Finally, we observe that

$$
\int_{\mathbb{R}}(1-\lambda) Z(\lambda) J(x) d F(x)-\int_{\mathbb{R}} \lambda(Z(1)-Z(\lambda)) J(x) d F(x)=(Z(\lambda)-\lambda Z(1)) \int_{\mathbb{R}} J(x) d F(x),
$$

and thus we have established Theorem 1.1.

Remark 2.1. (1) In our proof we have used an integration by parts in order to express our test statistic as a functional of the empirical process. A similar integration by parts technique was used by Dehling and Taqqu $(1989,1991)$. Note that in the present paper, we use a one-dimensional integration by parts formula, whereas Dehling and Taqqu use a two-dimensional integration by parts. The latter would not work here, as the kernel $1_{\{x \leq y\}}$ does not have locally bounded variation.

(2) As a corollary to Theorem 1.1, we obtain for fixed $\lambda \in[0,1]$ the asymptotic distribution of the Wilcoxon two-sample test, where the two samples are

$$
\begin{aligned}
& X_{1}, \ldots, X_{[n \lambda]} \\
& X_{[n \lambda]+1}, \ldots, X_{n} .
\end{aligned}
$$

After centering and normalization, the corresponding test statistic is

$$
U_{n}=\frac{1}{n d_{n}} \sum_{i=1}^{[n \lambda]} \sum_{j=[n \lambda]+1}^{n}\left(1_{\left\{X_{i} \leq X_{j}\right\}}-\int_{\mathbb{R}} F(x) d F(x)\right) .
$$

From Theorem 1.1 we obtain that $U_{n}$ converges in distribution to

$$
\frac{1}{m !}\left(Z_{m}(\lambda)-\lambda Z_{m}(1)\right) \int_{\mathbb{R}} J_{m}(x) d F(x)=\frac{1}{m !}\left((1-\lambda) Z_{m}(\lambda)-\lambda\left(Z_{m}(1)-Z_{m}(\lambda)\right)\right) \int_{\mathbb{R}} J_{m}(x) d F(x) .
$$

(3) A different two-sample problem arises when we observe samples from two independent 
long-range dependent processes $\left(X_{i}\right)_{i \geq 1}$ and $\left(X_{i}^{\prime}\right)_{i \geq 1}$ with identical joint distributions. In this case, the samples are

$$
\begin{aligned}
& X_{1}, \ldots, X_{[n \lambda]} \\
& X_{1}^{\prime}, \ldots, X_{n-[n \lambda]}^{\prime} .
\end{aligned}
$$

The normalized two-sample Wilcoxon test statistic for this case is

$$
U_{n}^{\prime}=\frac{1}{n d_{n}} \sum_{i=1}^{[n \lambda]} \sum_{j=1}^{n-[n \lambda]}\left(1_{\left\{X_{i} \leq X_{j}^{\prime}\right\}}-\int_{\mathbb{R}} F(x) d F(x)\right) .
$$

Following the proof of Theorem 1.1, and making the appropriate changes where needed, one can show that $U_{n}^{\prime}$ converges in distribution towards

$$
\left((1-\lambda) Z_{m}(\lambda)-\lambda Z_{m}^{\prime}(1-\lambda)\right) \int_{\mathbb{R}} J_{m}(x) d F(x),
$$

where $\left(Z_{m}^{\prime}(\lambda)\right)$ is an independent copy of $\left(Z_{m}(\lambda)\right)_{0 \leq \lambda \leq 1}$. Note that the limit distributions in the two models are different, as the joint distribution of $\left(Z_{m}(\lambda), Z_{m}(1)-Z_{m}(\lambda)\right)$ is different from that of $\left(Z_{m}(\lambda), Z_{m}^{\prime}(1-\lambda)\right)$. This is a result of the fact that the Hermite process does not have independent increments.

Observe that this is in contrast to the short-range dependent case. In this situation, the Wilcoxon two-sample test statistic has the same distribution in both models; see Dehling and Fried (2010). Roughly speaking, the dependence washes away in the limit for short-range dependence, but not for long-range dependence.

\section{Application}

In what follows, we will consider the model

$$
X_{i}=\mu_{i}+G\left(\xi_{i}\right), i=1, \ldots, n,
$$

where $\left(\xi_{i}\right)_{i \geq 1}$ is a mean-zero Gaussian process with $\operatorname{Var}\left(\xi_{i}\right)=1$ and autocovariance function $\rho(k)$ satisfying (1). We wish to test the hypothesis

$$
H: \mu_{1}=\ldots=\mu_{n}
$$

against the alternative

$$
A: \mu_{1}=\ldots=\mu_{k} \neq \mu_{k+1}=\ldots=\mu_{n} \text { for some } k \in\{1, \ldots, n-1\} .
$$

3.1. Wilcoxon-type rank test. The change-point test based on Wilcoxon's rank test will reject the null hypothesis for large values of

$$
W_{n}=\frac{1}{n d_{n}} \max _{1 \leq k \leq n-1}\left|\sum_{i=1}^{k} \sum_{j=k+1}^{n}\left(1_{\left\{X_{i} \leq X_{j}\right\}}-\frac{1}{2}\right)\right| .
$$

We first note an invariance property of the test statistic $W_{n}$.

Lemma 3.1. The test statistic $W_{n}$ is invariant under strictly monotone transformations of the data, i.e.

$$
\frac{1}{n d_{n}} \max _{1 \leq k \leq n-1}\left|\sum_{i=1}^{k} \sum_{j=k+1}^{n}\left(1_{\left\{G\left(X_{i}\right) \leq G\left(X_{j}\right)\right\}}-\frac{1}{2}\right)\right|=\frac{1}{n d_{n}} \max _{1 \leq k \leq n-1}\left|\sum_{i=1}^{k} \sum_{j=k+1}^{n}\left(1_{\left\{X_{i} \leq X_{j}\right\}}-\frac{1}{2}\right)\right|
$$

for all strictly monotone functions $G: \mathbb{R} \rightarrow \mathbb{R}$. 
Proof. If $G$ is strictly increasing, this is obvious, as $G\left(X_{i}\right) \leq G\left(X_{j}\right)$ if and only if $X_{i} \leq X_{j}$. If $G$ is strictly decreasing, $G\left(X_{i}\right) \leq G\left(X_{j}\right)$ if and only if $X_{j} \leq X_{i}$, and thus

$$
1_{\left\{G\left(X_{i}\right) \leq G\left(X_{j}\right)\right\}}=1-1_{\left\{X_{i} \leq X_{j}\right\}} .
$$

Hence we get

$$
\sum_{i=1}^{k} \sum_{j=k+1}^{n}\left(1_{\left\{G\left(X_{i}\right) \leq G\left(X_{j}\right)\right\}}-\frac{1}{2}\right)=-\sum_{i=1}^{k} \sum_{j=k+1}^{n}\left(1_{\left\{X_{i} \leq X_{j}\right\}}-\frac{1}{2}\right),
$$

and thus the lemma is proved.

Since $X_{i}=\mu_{i}+G\left(\xi_{i}\right)$, under the null hypothesis,

$$
W_{n}=\frac{1}{n d_{n}} \max _{1 \leq k \leq n-1}\left|\sum_{i=1}^{k} \sum_{j=k+1}^{n}\left(1_{\left\{G\left(\xi_{i}\right) \leq G\left(\xi_{j}\right)\right\}}-\frac{1}{2}\right)\right| .
$$

Thus, applying Theorem 1.1 and the continuous mapping theorem, under the null hypothesis, $W_{n}$ converges in distribution, as $n \rightarrow \infty$, to

$$
\sup _{0 \leq \lambda \leq 1}\left|\frac{Z_{m}(\lambda)}{m !}-\lambda \frac{Z_{m}(1)}{m !}\right|\left|\int_{\mathbb{R}} J_{m}(x) d F(x)\right| .
$$

In order to set critical values for the asymptotic test based on $W_{n}$, we need to calculate the distribution on the right-hand side.

In what follows, we will assume that $G$ is a strictly monotone function. In this case, combining (17) and Lemma 3.1 we get that

$$
W_{n}=\frac{1}{n d_{n}} \max _{1 \leq k \leq n-1}\left|\sum_{i=1}^{k} \sum_{j=k+1}^{n}\left(1_{\left\{\xi_{i} \leq \xi_{j}\right\}}-\frac{1}{2}\right)\right| .
$$

Note that in this case,

$$
J_{1}(x)=E\left(\xi 1_{\{\xi \leq x\}}\right)=\int_{-\infty}^{x} t \varphi(t) d t=-\varphi(x),
$$

where $\varphi(t)=\frac{1}{\sqrt{2 \pi}} e^{-t^{2} / 2}$ denotes the standard normal density function. In the last step, we have used the fact that $\varphi^{\prime}(t)=-t \varphi(t)$. Thus $J_{1}(x) \neq 0$ for all $x$ and hence the class of functions $\left\{1_{\{\xi \leq x\}}, x \in \mathbb{R}\right\}$ has Hermite rank $m=1$. Moreover, as $F$ is the normal distribution function we obtain

$$
\int_{\mathbb{R}} J_{1}(x) d F(x)=-\int_{R} \varphi(x) \varphi(x) d x=\int_{\mathbb{R}}-(\varphi(s))^{2} d s=-\frac{1}{2 \sqrt{\pi}} .
$$

We have thus proved the following theorem.

Theorem 3.2. Let $\left(\xi_{i}\right)_{i \geq 1}$ be a stationary Gaussian process with mean zero, variance 1 and autocovariance function as in (1) with $0<D<1$. Moreover, let $G: \mathbb{R} \rightarrow \mathbb{R}$ be a strictly monotone function and define $X_{i}=\mu_{i}+G\left(\xi_{i}\right)$. Then, under the null hypothesis $H$ : $\mu_{1}=\ldots=\mu_{n}$, the test statistic $W_{n}$, as defined in (16), converges in distribution towards

$$
\frac{1}{2 \sqrt{\pi}} \sup _{0 \leq \lambda \leq 1}\left|Z_{1}(\lambda)-\lambda Z_{1}(1)\right|
$$

where $\left(Z_{1}(\lambda)\right)_{\lambda \geq 0}$ denotes the standard fractional Brownian motion process with Hurst parameter $H=1-D / 2 \in(1 / 2,1)$. The normalizing constant in (16) satisfies $n d_{n} \sim$ $\left(\frac{L(n)}{(1-D)(1-D / 2)}\right)^{1 / 2} n^{2-D / 2}$. 

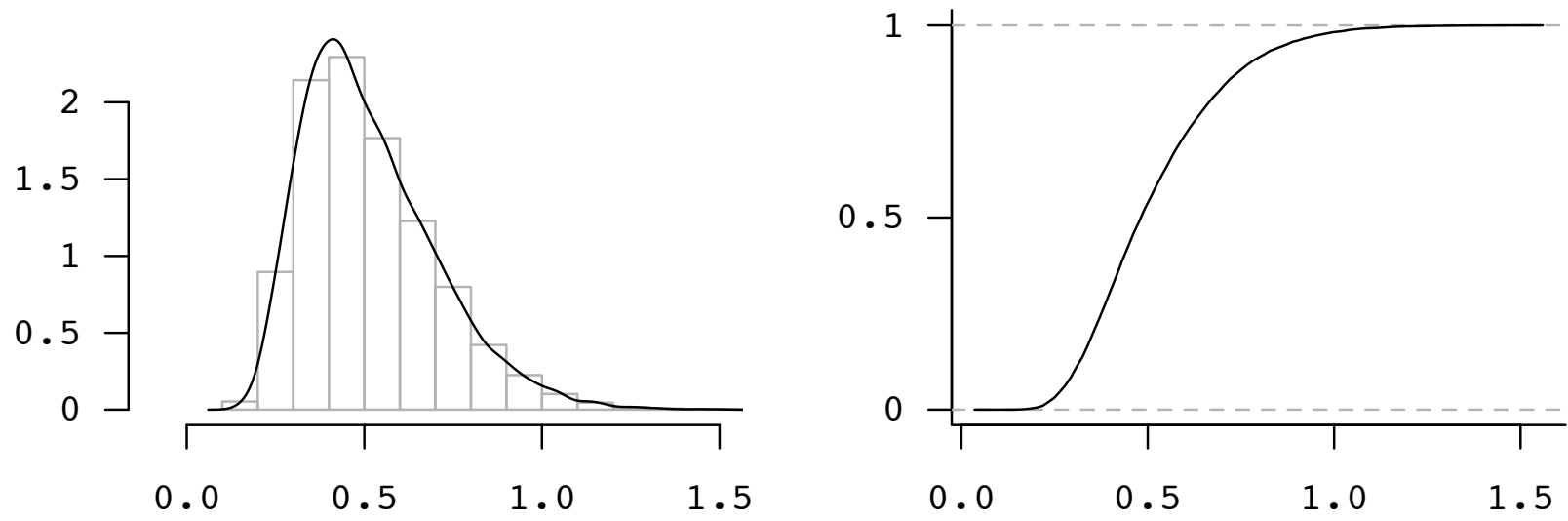

Figure 1. Probability density (left) and distribution function (right) of $\sup _{0 \leq \lambda \leq 1}|Z(\lambda)-\lambda Z(1)| ;$ calculations based on 10,000 simulation runs.

We have evaluated the distribution of $\sup _{0 \leq \lambda \leq 1}|Z(\lambda)-\lambda Z(1)|$ by simulating 10,000 realizations of a standard fractional Brownian motion $\left(Z^{(j)}(t)\right)_{0 \leq t \leq 1}, 1 \leq j \leq 10,000$, $t=\frac{i}{1000}, 0 \leq i \leq 1000$, using the fArma package in R. For each realization, we have calculated $M_{j}:=\max _{1 \leq i \leq 1000}\left|Z^{(j)}\left(\frac{i}{1000}\right)-\frac{i}{1000} Z^{(j)}(1)\right|$ as a numerical approximation to $\sup _{0 \leq \lambda \leq 1}\left|Z^{(j)}(\lambda)-\lambda Z^{(j)}(1)\right|$. The empirical distribution

$$
F_{M}(x):=\frac{1}{10,000} \#\left\{1 \leq j \leq 10,000: M_{j} \leq x\right\}
$$

of these 10,000 maxima was used as approximation to the distribution of $\sup _{0 \leq \lambda \leq 1} \mid Z(\lambda)-$ $\lambda Z(1) \mid$; see Figure 1 for the estimated probability density and the empirical distribution function, for the Hurst parameter $H=0.7$. We have calculated the corresponding upper $\alpha$-quantiles

$$
q_{\alpha}:=\inf \left\{x: F_{M}(x) \geq 1-\alpha\right\}
$$

for $H=0.6,0.7,0.9$; that is, $D=2-2 H=0.8,0.6,0.2$; see Table 1 .

\begin{tabular}{c|ccc}
$H / \alpha$ & 0.10 & 0.05 & 0.01 \\
\hline 0.6 & 0.98 & 1.10 & 1.34 \\
0.7 & 0.77 & 0.87 & 1.06 \\
0.9 & 0.38 & 0.44 & 0.54
\end{tabular}

TABLE 1. Upper $\alpha$-quantiles of $\sup _{0 \leq \lambda \leq 1}|Z(\lambda)-\lambda Z(1)|$, where $Z$ is a standard fBm, for different LRD parameters $H$, based on 10,000 repetitions.

3.2. A difference-of-means test. As an alternative, we also consider a test based on differences of means of the observations. We consider the test statistic

$$
D_{n}:=\max _{1 \leq k \leq n-1}\left|D_{k, n}\right|,
$$

where

$$
D_{k, n}:=\frac{1}{n d_{n}} \sum_{i=1}^{k} \sum_{j=k+1}^{n}\left(X_{i}-X_{j}\right)
$$


One would reject the null hypothesis $(15)$ if $D_{n}$ is large. To obtain the asymptotic distribution of the test statistic, we apply the functional non-central limit theorem of Taqqu (1979) and obtain that

$$
\begin{aligned}
\frac{1}{n d_{n}} \sum_{i=1}^{[n \lambda]} \sum_{j=[n \lambda]+1}^{n}\left(X_{i}-X_{j}\right) & =\frac{1}{n d_{n}}\left((n-[\lambda n]) \sum_{i=1}^{[n \lambda]} G\left(\xi_{i}\right)-[\lambda n] \sum_{j=[n \lambda]+1}^{n} G\left(\xi_{j}\right)\right) \\
& \stackrel{w}{\longrightarrow} a_{m}\left((1-\lambda) \frac{Z_{m}(\lambda)}{m !}-\lambda\left(\frac{Z_{m}(1)}{m !}-\frac{Z_{m}(\lambda)}{m !}\right)\right) \\
& =a_{m} \frac{1}{m !}\left(Z_{m}(\lambda)-\lambda Z_{m}(1)\right),
\end{aligned}
$$

where $m$ denotes the Hermite rank of $G(\xi)$ and where $a_{m}=E\left(G(\xi) H_{m}(\xi)\right)$ is the Hermite coefficient. Applying the continuous mapping theorem, we obtain the following theorem concerning the asymptotic distribution of the $D_{n}$.

Theorem 3.3. Let $\left(\xi_{i}\right)_{i \geq 1}$ be a stationary Gaussian process with mean zero, variance 1 and autocovariance function as in (1) with $0<D<1 / m$. Moreover, let $G: \mathbb{R} \rightarrow \mathbb{R}$ be a measurable function satisfying $E\left(G^{2}(\xi)\right)<\infty$ and define $X_{i}=\mu_{i}+G\left(\xi_{i}\right)$. Then, under the null hypothesis $H: \mu_{1}=\ldots=\mu_{n}$, the test statistic $D_{n}$, as defined in (20), converges in distribution towards

$$
\frac{\left|a_{m}\right|}{m !} \sup _{0 \leq \lambda \leq 1}\left|Z_{m}(\lambda)-\lambda Z_{m}(1)\right|
$$

where $\left(Z_{m}(\lambda)\right)$ denotes the $m$-th order Hermite process with Hurst parameter $H=1$ $D m / 2 \in(1 / 2,1)$.

Remark 3.4. For a strictly increasing function $G$, the Hermite rank is 1 , since

$$
E\left(G(\xi) H_{1}(\xi)\right)=\int_{\mathbb{R}} G(s) H_{1}(s) \varphi(s) d s=\int_{0}^{\infty} s \varphi(s)(G(s)-G(-s)) d s>0 .
$$

Similarly, for a stricly decreasing function $G$ we obtain $E\left(G(\xi) H_{1}(\xi)\right)<0$. Thus, in these cases the test statistic $D_{n}$ converges under the null hypothesis towards

$$
\left|a_{1}\right| \sup _{0 \leq \lambda \leq 1}\left|Z_{1}(\lambda)-\lambda Z_{1}(1)\right|
$$

where $Z_{1}$ is fractional Brownian motion with index $H=1-D / 2$. Note that in this case, up to a norming constant, the limit distribution of the difference-of-means test is the same as for the test based on Wilxocon's rank statistics.

\section{Difference-of-means test under fractional Gaussian noise}

We want to obtain a lower bound for the power of the difference-of-means test for fractional Gaussian noise, i.e. for the model

$$
X_{i}=\mu_{i}+\xi_{i}, \quad i=1, \ldots, n .
$$

While the distribution of the difference-of-means test statistic $D_{n}$, as defined in (20), is not explicitly known, one can calculate the exact distribution of

$$
D_{k, n}=\frac{1}{n d_{n}} \sum_{i=1}^{k} \sum_{j=k+1}^{n}\left(X_{i}-X_{j}\right)
$$


for the case of fractional Gaussian noise that we consider here. Recall that fractional Gaussian noise can be obtained by differencing fractional Brownian motion, that is

$$
\xi_{k}=B_{H}(k)-B_{H}(k-1)
$$

where $\left(B_{H}(t)\right)_{t \geq 0}$ is the standard fractional Brownian motion with Hurst parameter $H$, which we denoted $Z_{1}$. Its covariance is given in (6). The random variables $\xi_{k}$ have then mean zero, variance 1 and autocovariances

$$
\rho(k) \sim H(2 H-1) k^{2 H-2}=\left(1-\frac{D}{2}\right)(1-D) k^{-D}
$$

where $D=2-2 H$, and thus they have long-range dependence.

Consider the following alternative

$$
H_{\lambda, h}: E\left(X_{i}\right)=0 \text { for } i=1, \ldots,[n \lambda] \text { and } E\left(X_{i}\right)=h \text { for } i=[n \lambda]+1, \ldots, n \text {. }
$$

We shall compute the exact distribution of $D_{k, n}$ under $H_{\lambda, h}$ and thus obtain a lower bound for the power of $D_{n}$, since

$$
P\left(D_{n} \geq q_{\alpha}\right) \geq P\left(D_{[n \lambda], n} \geq q_{\alpha}\right)
$$

where $\left\{D_{n} \geq q_{\alpha}\right\}$ is the rejection region and $q_{\alpha}$ is given in (19).

First note that $d_{n}=n^{H}$ and thus $n d_{n}=n^{1+H}$, where $H$ is again the Hurst coefficient. $D_{k, n}$ has a normal distribution with mean

$$
E\left(D_{k, n}\right)=\frac{1}{n^{1+H}} \sum_{i=1}^{k} \sum_{j=k+1}^{n}\left(E\left(X_{i}\right)-E\left(X_{j}\right)\right) .
$$

Thus a small calculation shows that

$$
E\left(D_{k, n}\right)= \begin{cases}-\frac{1}{n^{1+H}} k(n-[n \lambda]) h & \text { if } k \leq[n \lambda] \\ -\frac{1}{n^{1+H}}(n-k)[n \lambda] h & \text { if } k \geq[n \lambda] .\end{cases}
$$

Note that $\max _{1 \leq k \leq n-1}\left|E\left(D_{k, n}\right)\right|=\left|E\left(D_{[n \lambda], n}\right)\right|=\frac{1}{n^{1+H}}(n-[n \lambda])[n \lambda] h \sim n^{1-H} \lambda(1-\lambda) h$. Since the variance of $D_{k, n}$ is not changed by the level shift, we get

$$
\begin{aligned}
\operatorname{Var}\left(D_{k, n}\right) & =\operatorname{Var}\left(\frac{1}{n^{1+H}} \sum_{i=1}^{k} \sum_{j=k+1}^{n}\left(\xi_{i}-\xi_{j}\right)\right) \\
& =\operatorname{Var}\left(\frac{1}{n^{1+H}}\left((n-k) \sum_{i=1}^{k} \xi_{i}-k \sum_{j=k+1}^{n} \xi_{j}\right)\right) \\
& =\operatorname{Var}\left(\frac{1}{n^{1+H}}\left((n-k) B_{H}(k)-k\left(B_{H}(n)-B_{H}(k)\right)\right)\right) \\
& =\operatorname{Var}\left(\frac{1}{n^{1+H}}\left(n B_{H}(k)-k B_{H}(n)\right)\right) .
\end{aligned}
$$


By the self-similarity of fractional Brownian motion, we finally get

$$
\begin{aligned}
\operatorname{Var}\left(D_{k, n}\right) & =\operatorname{Var}\left(B_{H}\left(\frac{k}{n}\right)-\frac{k}{n} B_{H}(1)\right) \\
& =\operatorname{Var}\left(B_{H}\left(\frac{k}{n}\right)\right)+\frac{k^{2}}{n^{2}} \operatorname{Var}\left(B_{H}(1)\right)-2 \frac{k}{n} \operatorname{Cov}\left(B_{H}\left(\frac{k}{n}\right), B_{H}(1)\right) \\
& =\left(\frac{k}{n}\right)^{2 H}+\frac{k^{2}}{n^{2}}-\frac{k}{n}\left(\left(\frac{k}{n}\right)^{2 H}+1-\left(1-\frac{k}{n}\right)^{2 H}\right) \\
& =\left(\frac{k}{n}\right)^{2 H}+\left(\frac{k}{n}\right)^{2}-\left(\frac{k}{n}\right)^{2 H+1}-\frac{k}{n}+\frac{k}{n}\left(1-\frac{k}{n}\right)^{2 H} \\
& =\left(\frac{k}{n}\right)^{2 H}\left(1-\frac{k}{n}\right)-\frac{k}{n}\left(1-\frac{k}{n}\right)+\frac{k}{n}\left(1-\frac{k}{n}\right)^{2 H} .
\end{aligned}
$$

Defining

$$
\sigma^{2}(\lambda)=\lambda^{2 H}(1-\lambda)-\lambda(1-\lambda)+\lambda(1-\lambda)^{2 H}
$$

we thus obtain

$$
\operatorname{Var}\left(D_{k, n}\right)=\sigma^{2}(k / n)
$$

The variance is maximal for $k=n / 2$, in which case we obtain

$$
\operatorname{Var}\left(D_{n / 2, n}\right)=\frac{1}{2^{2 H}}-\frac{1}{4} \approx 0.13
$$

The distribution of $D_{k, n}$ gives a lower bound for the power of the difference-of-means test at the alternative $H_{\lambda, h}$ considered above. We have

$$
\begin{aligned}
P\left(D_{n} \geq q_{\alpha}\right) \geq & P\left(\left|D_{[n \lambda], n}\right| \geq q_{\alpha}\right) \\
= & P\left(D_{[n \lambda], n} \leq-q_{\alpha}\right)+P\left(D_{[n \lambda], n} \geq q_{\alpha}\right) \\
= & P\left(\frac{D_{[n \lambda], n}+n^{1-H} \lambda(1-\lambda) h}{\sqrt{\sigma^{2}(\lambda)}} \leq \frac{-q_{\alpha}+n^{1-H} \lambda(1-\lambda) h}{\sqrt{\sigma^{2}(\lambda)}}\right) \\
& +P\left(\frac{D_{[n \lambda], n}+n^{1-H} \lambda(1-\lambda) h}{\sqrt{\sigma^{2}(\lambda)}} \geq \frac{q_{\alpha}+n^{1-H} \lambda(1-\lambda) h}{\sqrt{\sigma^{2}(\lambda)}}\right) \\
\approx & \Phi\left(\frac{-q_{\alpha}+n^{1-H} \lambda(1-\lambda) h}{\sqrt{\sigma^{2}(\lambda)}}\right)+1-\Phi\left(\frac{q_{\alpha}+n^{1-H} \lambda(1-\lambda) h}{\sqrt{\sigma^{2}(\lambda)}}\right),
\end{aligned}
$$

where $\Phi$ is the c.d.f. of a standard normal random variable. E.g., for $H=0.7, \lambda=\frac{1}{2}$, we get $q_{0.05}=0.87$ using Table 1 and thus

$$
P\left(D_{n} \geq q_{0.05}\right) \geq \Phi\left(\frac{-0.87+n^{0.3} h / 4}{\sqrt{\sigma^{2}(1 / 2)}}\right) \approx \Phi\left(-2.42+0.70 h n^{0.3}\right) .
$$

In this way, for sample size $n=500$ and level shift $h=1$ we get $\Phi(2.07) \approx .98$ as lower bound on the power of the difference-of-means test. For the same sample size, but $h=0.5$, we get the lower bound $\Phi(-0.18) \approx 0.43$. 


\section{Simulations}

In this section, we will present the results of a simulation study involving the Wilcoxon type rank test (16) and the difference-of-means test (20). We first investigate whether these tests reach their asymptotic level when applied in a finite sample setting, for sample sizes ranging from $n=10$ to $n=1,000$. Secondly, we compare the power of the two tests for sample size $n=500$ at various different alternatives

$$
A_{k}: \quad \mu_{1}=\ldots=\mu_{k} \neq \mu_{k+1}=\ldots=\mu_{n} .
$$

We let both the break point $k$ and the level shift $h:=\mu_{k+1}-\mu_{k}$ vary. Specifically, we choose $k=25,50,150,250$ and $h=0.5,1,2$.

As a basis for our simulations, we have taken realizations $\xi_{1}, \ldots, \xi_{n}$ of a fractional Gaussian noise (fGn) process with Hurst parameter $H$; respectively $D=2-2 H$, see (21) and (22). We have repeated each simulation 10,000 times.

5.1. Normally distributed data. In our first simulations, we took $G(t)=t$, so that $\left(X_{i}\right)_{i \geq 1}$ is fGn. $F$ is then the c.d.f. $\Phi$ of a standard normal random variable. In order to determine the critical values for the test statistics $W_{n}$ and $D_{n}$, we have applied Theorem 3.2 and Theorem 3.3. Since $G$ is strictly increasing, Theorem 3.2 yields that, under the null hypothesis, $W_{n}$ has approximately the same distribution as

$$
\frac{1}{2 \sqrt{\pi}} \sup _{0 \leq \lambda \leq 1}\left|Z_{1}(\lambda)-\lambda Z_{1}(1)\right| .
$$

Since $G$ is the first Hermite polynomial, its Hermite rank is $m=1$ and the associated Hermite coefficient is $a_{1}=1$. Hence, Theorem 3.3 yields that, under the null hypothesis, the test statistic $D_{n}$ has approximately the same distribution as

$$
\sup _{\lambda \in[0,1]}\left|Z_{1}(\lambda)-\lambda Z_{1}(1)\right|
$$

We have calculated asymptotic critical values for both tests by using the upper 5\%-quantiles of $\sup _{\lambda \in[0,1]}\left|Z_{1}(\lambda)-\lambda Z_{1}(1)\right|$, as given in Table 1. Thus the Wilcoxon-type test rejected the

\begin{tabular}{|c|c|c|c|c|c|c|c|}
\hline$n / H$ & 0.6 & 0.7 & 0.9 & $n / H$ & 0.6 & 0.7 & 0.9 \\
\hline 10 & 0.024 & 0.031 & 0.038 & 10 & 0.013 & 0.026 & 0.326 \\
\hline 50 & 0.039 & 0.042 & 0.047 & 50 & 0.042 & 0.050 & 0.167 \\
\hline 100 & 0.042 & 0.046 & 0.044 & 100 & 0.044 & 0.051 & 0.140 \\
\hline 500 & 0.047 & 0.052 & 0.049 & 500 & 0.051 & 0.052 & 0.100 \\
\hline 1000 & 0.047 & 0.052 & 0.053 & 1000 & 0.051 & 0.054 & 0.095 \\
\hline
\end{tabular}
null hypothesis when $W_{n} \geq \frac{1}{2 \sqrt{\pi}} q_{\alpha}$, while the difference-of-means test rejected when $D_{n} \geq q_{\alpha}$, where $q_{\alpha}$ is given in (19).

TABLE 2. Level of "difference-of-means" test (left) and level of "Wilcoxontype" test (right) for fGn time series with LRD parameter $H$; 10, 000 simulation runs. Both tests have asymptotically level $5 \%$.

We have checked whether the tests reach their asymptotic level of $5 \%$ and counted the number of rejections of the null hypothesis in 10,000 simulations, where the null hypothesis was true. We see in Table 2 that both tests perform well already for moderate sample sizes of $n=50$, with the notable exception of the Wilcoxon-type test when $H=0.9$, i.e. when we have very strong dependence. In that case, convergence in Theorem 3.2 appears to be very 

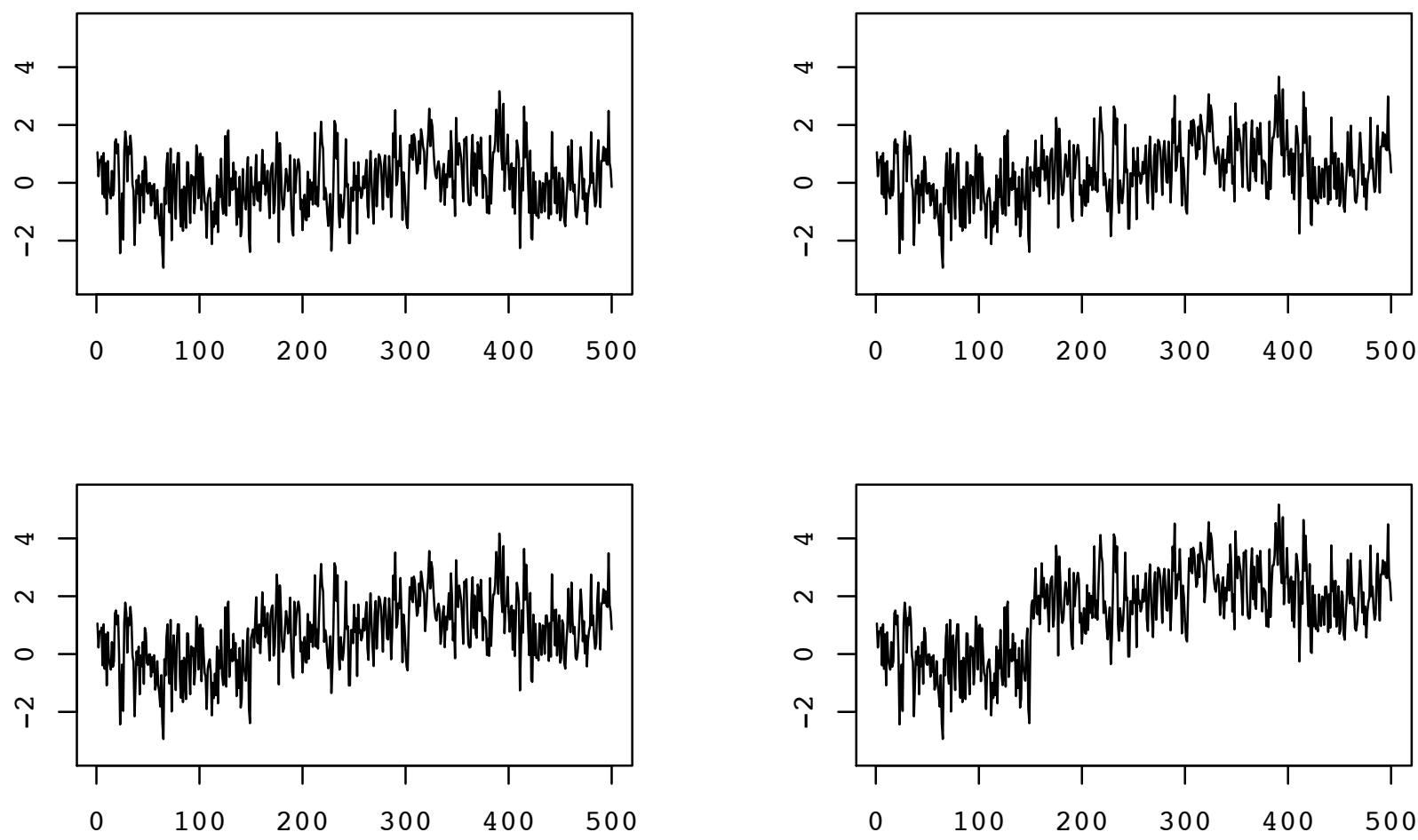

FigURE 2. fGn without breaks (top left) and with a jump after observation 150 (this is $[\lambda n]$ with $\lambda=0.3$ ) of height $h=0.5$ (top right), $h=1$ (bottom left) and $h=2$ (bottom right).

slow so that the asymptotic critical values are misleading when applied in a finite sample setting.

In order to analyze how well the tests detect break points, we have introduced a level shift $h$ at time $[n \lambda]$, i.e. we consider the time series

$$
X_{i}= \begin{cases}\xi_{i} & \text { for } i=1, \ldots,[n \lambda] \\ \xi_{i}+h & \text { for } i=[n \lambda]+1, \ldots, n .\end{cases}
$$

We have done this for several choices of $\lambda$ and $h$, for sample size $n=500$. As Table 3 shows, both tests detect breaks very well - and the better, the larger the level shift is and the more in the middle the shift takes place. When the break occurs in the middle, both tests perform equally well. Breaks at the beginning are better detected by the difference-of-means test.

\begin{tabular}{c|cccc}
$h / \lambda$ & 0.05 & 0.1 & 0.3 & 0.5 \\
\hline 0.5 & 0.060 & 0.090 & 0.388 & 0.524 \\
1 & 0.090 & 0.254 & 0.952 & 0.985 \\
2 & 0.261 & 0.965 & 1.000 & 1.000
\end{tabular}

\begin{tabular}{c|cccc}
$h / \lambda$ & 0.05 & 0.1 & 0.3 & 0.5 \\
\hline 0.5 & 0.058 & 0.086 & 0.386 & 0.525 \\
1 & 0.077 & 0.184 & 0.948 & 0.985 \\
2 & 0.119 & 0.763 & 1.000 & 1.000
\end{tabular}

TABLE 3. Power of "difference-of-means" test (left) and power of "Wilcoxontype" test (right) for $n=500$ observations of fGn with LRD parameter $H=$ 0.7, different break points $[\lambda n]$ and different level shifts $h$. Both tests have asymptotically level 5\%. The calculations are based on 10,000 simulation runs. 
5.2. Heavy-Tailed Data. In the second set of simulations, we took Pareto distributed data. Note that the $\operatorname{Pareto}(\beta, k)$ distribution has distribution function

$$
F(x)= \begin{cases}1-\left(\frac{k}{x}\right)^{\beta} & \text { if } x \geq k \\ 0 & \text { else }\end{cases}
$$

where the scale parameter $k$ is the smallest possible value for $x$ and where $\beta$ is a shape parameter. The Pareto distribution has finite expected value when $\beta>1$ and finite variance when $\beta>2$. The expected value and the variance are given by

$$
\begin{aligned}
\mu & =E(X)=\frac{\beta k}{\beta-1}, \quad \beta>1 \\
\sigma^{2} & =\operatorname{Var}(X)=\frac{\beta k^{2}}{(\beta-1)^{2}(\beta-2)}, \quad \beta>2 .
\end{aligned}
$$

In order to obtain $\operatorname{Pareto}(\beta, k)$-distributed $X=G(\xi)$, we take $G$ to be the quantile transform, i.e. $G(t)=k(\Phi(t))^{-1 / \beta}$ where $\Phi$ denotes the standard normal distribution function, so that for $x \geq k$

$$
P\left(X_{i} \geq x\right)=P\left(G\left(\xi_{i}\right) \geq x\right)=P\left(\left(\Phi\left(\xi_{i}\right)\right)^{-1 / \beta} \geq \frac{x}{k}\right)=P\left(\xi_{i} \leq \Phi^{-1}\left(\left(\frac{k}{x}\right)^{\beta}\right)\right)=\left(\frac{k}{x}\right)^{\beta} .
$$

Since we want the $X_{i}$ to be centered, we will in fact take

$$
G(t)=k(\Phi(t))^{-1 / \beta}-\frac{\beta k}{\beta-1} .
$$

If we want $X_{i}$ to be standardized to have mean 0 and variance 1 , we will consider $Z=$ $(X-\mu) / \sigma$ and take

$$
G(t)=\left(\frac{\beta k^{2}}{(\beta-1)^{2}(\beta-2)}\right)^{-1 / 2}\left(k(\Phi(t))^{-1 / \beta}-\frac{\beta k}{\beta-1}\right)
$$

The corresponding distribution function of $Z$ is then

$$
F_{Z}(z)= \begin{cases}1-\left(\frac{k}{\sigma z+\mu}\right)^{\beta} & \text { if } z \geq \frac{k-\mu}{\sigma} \\ 0 & \text { else. }\end{cases}
$$

and its density function is

$$
f_{Z}(z)= \begin{cases}k^{\beta} \beta \sigma(\sigma z+\mu)^{-\beta-1} & \text { if } z \geq \frac{k-\mu}{\sigma} \\ 0 & \text { else }\end{cases}
$$

Pareto(3, 1) Data: We first performed simulations with Pareto $(3,1)$ data, i.e. heavy-tailed data with finite variance. In this case, $\beta=3, k=1$ and we have $E(X)=\frac{3}{2}$ and $\operatorname{Var}(X)=\frac{3}{4}$. For a better comparison with the simulations involving fractional Gaussian noise, we also standardize the data, i.e. we consider (see (24)),

$$
G(t)=\frac{1}{\sqrt{3 / 4}}\left((\Phi(t))^{-1 / 3}-\frac{3}{2}\right) .
$$

The probability density function of the standardized $X$ is given by (see (26)),

$$
f(x)= \begin{cases}3 \sqrt{\frac{3}{4}}\left(\sqrt{\frac{3}{4}} x+\frac{3}{2}\right)^{-4} & \text { if } x \geq-\sqrt{\frac{1}{3}} \\ 0 & \text { else. }\end{cases}
$$


$G$ is again strictly decreasing, and by the above results, the Hermite rank of the class of functions $\left\{I_{\left\{G\left(\xi_{i}\right) \leq x\right\}}-F(x), x \in \mathbb{R}\right\}$ is $m=1$, the Hermite rank of $G$ itself is $m=1$ and $\left|\int_{\mathbb{R}} J_{1}(x) d F(x)\right|=(2 \sqrt{\pi})^{-1}$, see (18). Numerical integration yields

$$
a_{1}=E[\xi G(\xi)]=\sqrt{\frac{4}{3}} \int_{-\infty}^{\infty} s \Phi(s)^{-1 / 3} \varphi(s) d s \approx-0.6784 .
$$

Table 4 shows the observed level of the tests, for various sample sizes and various Hurst parameters. For sample sizes up to $n=1,000$, the "difference-of-means" test has level larger than 10\%. We conjecture that this is due to the slow convergence in Theorem 3.3. This conjecture is supported by the outcomes of simulations with sample sizes $n=2,000$ and $n=10,000 ;$ see Table 4 .

\begin{tabular}{|c|c|c|c|c|c|c|c|}
\hline$n / H$ & 0.6 & 0.7 & 0.9 & & & & \\
\hline 10 & 0.104 & 0.109 & 0.117 & $n / H$ & 0.6 & 0.7 & 0.9 \\
\hline 50 & 0.138 & 0.127 & 0.126 & 10 & 0.013 & 0.026 & 0.326 \\
\hline 100 & 0.145 & 0.125 & 0.126 & 50 & 0.042 & 0.050 & 0.167 \\
\hline 500 & 0.140 & 0.103 & 0.119 & 100 & 0.044 & 0.051 & 0.140 \\
\hline 1000 & 0.131 & 0.101 & 0.123 & 500 & 0.051 & 0.052 & 0.100 \\
\hline 2000 & 0.120 & 0.086 & 0.115 & 1000 & 0.051 & 0.054 & 0.095 \\
\hline 10000 & 0.106 & 0.069 & 0.101 & & & & \\
\hline
\end{tabular}

TABLE 4. Level of "difference-of-means" test (left) and level of Wilcoxon-type test (right) for standardised Pareto(3,1)-transformed fGn with LRD parameter $H ; 10,000$ simulation runs. Both tests have asymptotically level 5\%.

Table 5 gives the observed power of the "difference-of-means" test and the Wilcoxon-type test, for sample size $n=500$ and various values of the break points and height of level shift. The results show that the Wilcoxon-type test has larger power than the "difference-ofmeans" test for small level shift $h$, but that the "difference-of-means" test outperforms the Wilcoxon type test for larger level shifts.

\begin{tabular}{|c|c|c|c|c|c|c|c|c|c|}
\hline$h / \lambda$ & 0.05 & 0.1 & 0.3 & 0.5 & $h / \lambda$ & 0.05 & 0.1 & 0.3 & 0.5 \\
\hline 0.5 & 0.116 & 0.177 & 0.756 & 0.864 & 0.5 & 0.088 & 0.294 & 0.983 & 0.99 \\
\hline 1 & 0.177 & 0.693 & 0.998 & 1.000 & 1 & 0.1 & 0.655 & 1.000 & 1.00 \\
\hline 2 & 0.815 & 0.998 & 1.000 & 1.000 & 2 & 0.138 & 0.944 & 1.000 & 1.00 \\
\hline
\end{tabular}

TABLE 5. Power of "difference-of-means" test (left) and power of "Wilcoxontype" test (right) for $n=500$ observations of standardised Pareto(3,1)transformed fGn with LRD parameter $H=0.7$, different break points $[\lambda n]$ and different level shifts $h$. Both tests have asymptotically level $5 \%$. The calculations are based on 10,000 simulation runs.

However, the above comparison is not really meaningful, since the "difference-of-means" test has a realized level of approximately $10 \%$ while the Wilcoxon-type test has level close to $5 \%$; see Table 4. Thus we have calculated the finite sample 5\%-quantiles of the distribution of the "difference-of-means" test, using a Monte-Carlo simulation; see Table 6. For example, for $n=500$ and $H=0.7$, the corresponding critical value is 0.70 . Thus we reject the null hypothesis of no break point if the "difference-of-means" test statistic is greater than 0.70 . 


\begin{tabular}{c|cccccccc}
$H / n$ & 10 & 50 & 100 & 500 & 1,000 & 2,000 & 10,000 & $\infty$ \\
\hline 0.6 & 1.02 & 1.04 & 1.02 & 0.93 & 0.90 & 0.88 & 0.85 & 0.75 \\
0.7 & 0.84 & 0.82 & 0.79 & 0.70 & 0.68 & 0.65 & 0.62 & 0.59 \\
0.9 & 0.47 & 0.47 & 0.44 & 0.43 & 0.42 & 0.41 & 0.38 & 0.30
\end{tabular}

TABLE 6. 5\%-quantiles of the finite sample distribution of the "difference-ofmeans" test under the null hypothesis for Pareto(3,1)-transformed fGn with different LRD parameter $H$ and different sample sizes $n$. The calculations are based on 10,000 simulation runs.

The value of 0.70 should be contrasted to the asymptotic $(n=\infty)$ value of 0.59 . To obtain the asymptotic critical values, we proceeded as follows: according to Theorem 3.3, the asymptotic distribution, under the null hypothesis, of the test statistic $D_{n}$ equals the distribution of

$$
\left|a_{1}\right| \sup _{0 \leq \lambda \leq 1}|Z(\lambda)-\lambda Z(1)| .
$$

Thus the asymptotic upper $\alpha$-quantiles of $D_{n}$ can be calculated as $\left|a_{1}\right| q_{\alpha}$, where $q_{\alpha}$ is the upper $\alpha$-quantile of the distribution of $\sup _{0 \leq \lambda \leq 1}|Z(\lambda)-\lambda Z(1)|$, as tabulated in Table 1 .

\begin{tabular}{c|cccc}
$h / \lambda$ & 0.05 & 0.1 & 0.3 & 0.5 \\
\hline 0.5 & 0.053 & 0.078 & 0.566 & 0.733 \\
1 & 0.078 & 0.379 & 0.994 & 0.999 \\
2 & 0.423 & 0.994 & 1.000 & 1.000
\end{tabular}

TABle 7. Power of the "difference-of-means" test, based on the finite sample quantiles, for $n=500$ observations of Pareto(3,1)-transformed fGn with LRD parameter $H=0.7$, different break points $[\lambda n]$ and different level shifts $h$ (left). The calculations are based on 10,000 simulation runs.

We have then calculated the power of the "difference-of-means" test through simulation, with $n=500, H=0.7$ and the finite sample quantile critical value of 0.70 rather than the asymptotic value of 0.59 (see Table 6). Table 7 shows the power of the test. We can now compare the results of the Wilcoxon-type test given in the right-hand side of Table 5 with the finite sample "difference-of-means" test results given in Table 7 . We see that the Wilcoxontype test has better power than the "difference-of-means" test, except for large level shifts at an early time. Such changes are detected more often by the "difference-of-means" test.

Pareto $(2,1)$ Data: We now choose $k=1$ and $\beta=2$, so that the $X$ have finite expectation, but infinite variance. In order to have centered data, we take $G$ as in (23), i.e.

$$
G(t)=\frac{1}{\sqrt{\Phi(t)}}-2 .
$$

We will now compare both tests, i.e. the Wilcoxon-type test and the difference of means test, although the latter can strictly speaking not be applied because it requires data with finite variance, respectively $G \in L_{2}(\mathbb{R}, \mathcal{N})$. By Theorem 3.2, under the null hypothesis of no change, the Wilcoxon-type test statistic $W_{n}$ converges in distribution towards

$$
\frac{1}{2 \sqrt{\pi}} \sup _{0 \leq \lambda \leq 1}\left|Z_{1}(\lambda)-\lambda Z_{1}(1)\right| .
$$




\begin{tabular}{|c|c|c|c|c|c|c|c|}
\hline$n / H$ & 0.6 & 0.7 & 0.9 & $n / H$ & 0.6 & 0.7 & 0.9 \\
\hline 10 & 0.104 & 0.104 & 0.107 & 10 & 0.013 & 0.026 & 0.326 \\
\hline 50 & 0.159 & 0.138 & 0.120 & 50 & 0.042 & 0.050 & 0.167 \\
\hline 100 & 0.181 & 0.151 & 0.122 & 100 & 0.044 & 0.051 & 0.140 \\
\hline 500 & 0.223 & 0.148 & 0.124 & 500 & 0.051 & 0.052 & 0.100 \\
\hline 1000 & 0.232 & 0.151 & 0.130 & 1000 & 0.051 & 0.054 & 0.095 \\
\hline
\end{tabular}

TABLE 8. Level of "difference-of-means" test (left) and level of "Wilcoxontype" test (right) for Pareto(2,1)-transformed fGn with LRD parameter $H$; 10, 000 simuation runs.

In fact, as a consequence of Lemma 3.1, even the finite sample distribution of $W_{n}$ is the same as for normally distributed data. Table 8 gives the measured level of the Wilcoxon-type test (the asymptotic level is 5\%) and Table 9 suggests it has good power, especially for small shifts in the middle of the observations.

\begin{tabular}{|c|c|c|c|c|c|c|c|c|c|}
\hline$h / \lambda$ & 0.05 & 0.1 & 0.3 & 0.5 & $h / \lambda$ & 0.05 & 0.1 & 0.3 & 0.5 \\
\hline 0.5 & 0.148 & 0.156 & 0.272 & 0.350 & 0.5 & 0.075 & 0.180 & 0.878 & 0.960 \\
\hline 1 & 0.156 & 0.200 & 0.741 & 0.8 & 1 & 0.097 & 0.401 & 0.997 & 1.000 \\
\hline 2 & 0.199 & 0.651 & 0.996 & 0.999 & 2 & 0.122 & 0.744 & 1.000 & 1.000 \\
\hline
\end{tabular}

TABLE 9. Power of "difference-of-means" test (left) and power of "Wilcoxontype" test (right) for $n=500$ observations of Pareto(2,1)-transformed fGn with LRD parameter $H=0.7$, different break points $[\lambda n]$ and different level shifts $h$. The calculations are based on 10,000 simulation runs.

Let us now consider the difference-of-means test. Note that, strictly speaking, Theorem 3.3 cannot be applied in the case of the Pareto data with $\beta=2$ because it requires the variance of the data to be finite. It is interesting nevertheless to use the asymptotic test suggested by Theorem 3.3. Since $G$ is strictly monotone, the Hermite rank of $G$ is $m=1$ as well, by the remark following Theorem 3.3. Using numerical integration, we have calculated

$$
a_{1}=E[\xi G(\xi)]=\int_{-\infty}^{\infty} s\left(\Phi(s)^{-1 / 2}-2\right) \varphi(s) d s=\int_{-\infty}^{\infty} s \Phi(s)^{-1 / 2} \varphi(s) d s \approx-1.40861 .
$$

We clearly see in Table 8 that the difference-of-means test very often falsely rejects the null hypothesis, that is detects breaks where there are none, while the Wilcoxon-type test is robust. Table 9 shows that both tests have good power, but again, the Wilcoxon tests is clearly better, especially for small shifts in the middle of the observations.

\section{REFERENCES}

[1] Jaromír Antoch, Marie Hušková, Alicja Janic and Teresa Ledwina (2008): Data driven rank tests for the change point problem. Metrika 68, 1-15.

[2] Alexander Aue, Siegfried Hörmann, Lajos Horváth and Matthew Reimherr (2009): Break detection in the covariance structure of multivariate time series. The Annals of Statistics 37, 4046-4087.

[3] István Berkes, Lajos Horváth, Piotr Kokoszka and Qi-Man Shao (2006): On discriminating between long-range dependence and changes in the mean. The Annals of Statistics 34, 1140-1165.

[4] Miklós Csörgő and Lajos Horváth (1997): Limit Theorems in Change-Point Analysis. J. Wiley \& Sons, Chichester. 
[5] Herold Dehling and Roland Fried (2010): Asymptotic Distribution of Two-Sample Empirical U-Quantiles with Applications to Robust Tests for Structural Change. SFB 823 Discussion Paper $43 / 2010$.

[6] Herold Dehling and Murad S. Taqqu (1989): The empirical process of some long-range dependent sequences with an application to U-statistics. The Annals of Statistics 17, 1767-1783.

[7] Herold Dehling and Murad S. Taqqu (1991): Bivariate symmetric statistics of long-range dependent observations. Journal of Statistical Planning and Inference 28, 153-165.

[8] Walter Krämer, Philipp Sibbertsen and Christian Kleiber (2002): Long memory versus structural change in financial time series. Allgemeines Statistisches Archiv 86, 83-96.

[9] Erich Lehmann (1975): Nonparametrics: Statistical Methods Based on Ranks. Holden-Day, San Francisco.

[10] Shiqing Ling (2007): Testing for change points in time series models and limiting theorems for NED sequences. The Annals of Statistics 35, 1213-1227.

[11] Galen R. Shorack and Jon A. Wellner (1986): Empirical Processes with Applications to Statistics. John Wiley \& Sons, New York.

[12] Murad S. TAqQU (1979): Convergence of integrated processes of arbitrary Hermite rank. Zeitschrift für Wahrscheinlichkeitstheorie und verwandte Gebiete 50, 53-83.

[13] Lihong Wang (2008): Change-Point Detection with Rank Statistics in Long-Memory Time-Series Models. Australian 83 New Zealand Journal of Statistics 50, 241-256.

[14] Dominik Wied, Walter Krämer and Herold Dehling (2011): Testing for a change in correlation at an unknown point in time using an extended functional delta method. Econometric Theory (to appear).

Fakultät für Mathematik, Ruhr-Universität Bochum, 44780 Bochum, Germany

E-mail address: herold.dehling@rub.de

Fakultät für Mathematik, Ruhr-Universität Bochum, 44780 Bochum, Germany

E-mail address: aeneas.rooch@rub.de

Department of Mathematics, 111 Cummington St., Boston University, Boston MA 02215

E-mail address: murad@bu.edu 


